\title{
ARTIGO CIENTÍEICO \\ Mimosa L. (Fabaceae: Caesalpinioideae) no herbário Instituto Agronômico do Norte da Embrapa Amazônia Oriental
}

\section{Mimosa L. (Fabaceae: Caesalpinioideae) in the herbarium Northern Agronomic Institute of Embrapa Eastern Amazonia}

\author{
Milton Garcia Costal, Lorena Lisboa Araujo ${ }^{2}$, Helena Joseane Raiol Souza ${ }^{3}$, Sebastião Ribeiro Xavier Júnior ${ }^{4}$, Wanderson \\ Luís da Silva e Silva ${ }^{5}$
}

\begin{abstract}
Resumo: As coleções biológicas são testemunhos da biodiversidade e instrumento imprescindível para vários estudos e, neste contexto, surge o Herbário IAN da Embrapa Amazônia Oriental, indexado pelo Index Herbariorum sob o acrônimo IAN, pois na época de sua criação (1945) a empresa chamava-se Instituto Agronômico do Norte. O IAN contém uma ampla diversidade de amostras do gênero Mimosa L., que apresenta notável potencial econômico por conter várias aplicações na medicina popular, recuperação de áreas degradadas, serviços ambientais, alimentação animal, madeireira e outras utilidades. Este estudo tem como objetivo realizar o levantamento, informatização e disponibilização do gênero Mimosa L. no acervo do herbário IAN da Embrapa Amazônia Oriental, destacando a relevância da coleção e diversidade de amostras existentes. Os dados sobre o gênero foram extraídos do banco de dados do Herbário IAN com auxílio do software BRAHMS (Botanical Research And Management System) e confrontados com as informações contidas nas exsicatas, onde foi possível corrigir e acrescentar dados e imagens de amostras do acervo que não constavam no banco de dados. Após a verificação e correção dos dados e imagens, a coleção de Mimosa foi disponibilizada via internet. Foram obtidas 683 amostras do gênero Mimosa, com presença de 176 espécies coletadas em diversos locais, sendo o estado do Pará o mais representativo, ressaltando que a quantidade de espécies encontradas corresponde a 34,5\% de todas as espécies de Mimosa no mundo. Acerca da informatização, constatou-se que cerca de $93 \%$ da coleção de Mimosa do herbário IAN está informatizado com suas respectivas imagens devidamente digitalizadas e, 86\% de toda a coleção de Mimosa disponibilizado no Herbário Virtual IAN. O trabalho também ressalta a importância do processo de informatização e disponibilização das coleções, pois proporcionam agilidade e acessibilidade para a comunidade científica e à sociedade em geral..
\end{abstract}

Palavras-chave: Levantamento; Informatização; Disponibilização; Biodiversidade.

Abstract: The biological collections are evidence of the biodiversity and requisit instrument for several studies and, in this context, the Herbarium of Embrapa Amazônia Oriental appears, indexed by the Index Herbariorum under the acronym IAN, because at the time of its creation (1945) the company was called Instituto Agronômico do Norte. The IAN contains a wide diversity of gender Mimosa L., which shows notable economical potential by having a lot of applications in folk medicine, recovery of degraded areas, environmental services, animal food, timber and other utilities. This present study aims to realize the database collection, informatization and availability of Mimosa L. genus, in the herbarium IAN collection of Embrapa Oriental Amazon, highlighting the relevance of the collection and the diversity of existent samples. The data which contains the genus were extracted from the Herbarium IAN database with the software BRAHMS (Botanical Research And Management System) and compared with the data contained in the exsiccates, were it was possible to repair, add data and images that are not found in the database. After the verification and correction of the data and images, the Mimosa collection was made available via internet. So, there were obtained 683 gender Mimosa samples, with presence of 176 species collected in several places, and the estate of Pará being the most representative, emphasizing that the amount of species found corresponds to $34.5 \%$ of all the Mimosa species in the world. About computerization, it was found that around $93 \%$ of Mimosa from Herbarium IAN is computerized and with its respective scanned images, $86 \%$ of the entire Mimosa collection is available in the IAN virtual herbarium. The present study also points out the importance of the process of computerization and availability of collections, as they provide agility and accessibility for the scientific community and society in general.

Key words: Survey; Computerization; Available; Biodiversity

\footnotetext{
*Autor para correspondência

Recebido para publicação em 11/12/2017; aprovado em 05/06/2018

${ }^{1}$ Graduando de Agronomia, Universidade Federal Rural da Amazônia , Capitão Poço, Pará; (91) 99637-1450, miltongarciacosta.2010@ gmail.com

${ }^{2}$ Graduanda de Ciências Biológicas, Universidade da Amazônia, lorenalisboaa8@ gmail.com

${ }^{3}$ Especialista em Oleoquímica, EMBRAPA, Amazônia Oriental. helena.souza@embrapa.br

${ }^{4}$ Especialista em Perícia e avaliação de impactos ambientais, EMBRAPA, Amazônia Oriental. sebastiao.xavier@embrapa.br

${ }^{5}$ Consultor do Inventário Florestal Nacional, Serviço Florestal Nacional. wl.silvasilva@ gmail.com
} 


\section{INTRODUÇÃO}

Mimosa L. pertence à família FabaceaeCaesalpinioideae que compreende cerca de 490 a 510 espécies sendo elas distribuídas na região Palaeotropical (subcontinente indiano), Novo Mundo (América Central e do Sul), México e Estados Unidos (LEWIS et al., 2005; LPWG, 2017). No Brasil, a ocorrência do gênero se faz presente em todas as regiões, sendo este, entre as Angiospermas, o segundo mais rico do país, tendo maior diversidade no Cerrado e na Caatinga (SILVA, 2013; BFG, 2015; MORIM, 2015).

O gênero revela uma diversidade evidente em relação à morfologia, mas apresenta fruto do tipo craspédio com poucas exceções (BORGES, 2014). As suas características morfológicas variam, entre caules armados ou não; indumentos diversos; folhas bipinadas, foliólulos sésseis, geralmente o primeiro par de cada pina diferenciado; flores com três, cinco ou seis pétalas, podendo ser isostêmones ou diplostêmones, filetes cremes, róseos ou amarelos, livres ou monadelfos; sementes livres, não carnosas, lentiformes, geralmente marrons e com pleurograma presente (DUTRA, 2009; BORGES, 2010).

Apresentam-se nos domínios fitogeográficos de florestas tropicais e subtropicais, savanas, campos e desertos, e podem ser encontrados em formas de árvores, arbustos, ervas, liana e subarbustos; são utilizadas como plantas ornamentais, cercas vivas, forragem, adubação verde, plantas para sombreamento, lenha e em medicamentos utilizados para cicatrização de queimaduras, picadas de alguns animais silvestres e como sedativo natural, porém, muitas espécies são daninhas prejudicando terras agrícolas (LEWIS et al., 2005; NASCIMENTO et al., 2012).

Possui elevado potencial para Recuperação de Áreas Degradadas (RAD), pois tem boa adaptação em solos acidentados; apresenta vantagens recíprocas em consorciação e algumas espécies são pioneiras em RAD e reflorestamento. Dentre elas a Mimosa caesalpiniifolia Benth que evidencia um alto potencial para regeneração, pois quando plantada em consórcio com outros vegetais, beneficia espécies com maior relevância (CARVALHO, 2007). A diversidade de espécies utilizadas em RAD vem aumentando com frequência por serem pioneiras e transformadoras de locais impactados, tais como, margem de estradas, rios e clareiras de matas, o que tornaram as espécies do gênero Mimosa notáveis e com potencialidades para regeneração de áreas degradadas (DOURADO, 2013).

Outras espécies que têm aptidão para recuperação de solos são as Mimosa flocculosa Burkart e Mimosa bimucronata (DC.) Kuntze que auxiliam na restauração de áreas degradas por exploração mineral e terrenos planados; são características destas o processo de fixação de nitrogênio, apresentando uma decomposição de biomassa no solo eficaz que contribui para uma rápida regeneração, gerando aumento da diversidade local em terrenos úmidos e rochosos, e ainda, pode ser utilizada para controlar erosões no solo (CARVALHO, 1994, 2004). M. bimucronata (DC.) também é utilizada na arborização urbana e cercas vivas (COUTINHO, 2009), bem como, oferece utilidade para tratamentos de patologias, em que os brotos desta espécie são aproveitados para tratar asma, bronquite e febres (CARVALHO, 1994; CORADIN, 2011).
Mimosa caesalpiniifolia Benth, pode ser utilizada como cerca viva ou arborização urbana e para fins madeireiros voltados à produção de adesivos, fabricação de chapas de madeiras aglomeradas, estacas, mourões, varas e produção de lenha (COUTINHO, 2009). Mimosa scabrella Benth. possui o crescimento rápido e alta densidade, por este motivo é cultivada frequentemente por agricultores e produtores rurais, para fins de produção de lenha e carvão, e por apresentar um alto índice de absorção de carbono, sendo possível a prestação de serviços ambientais para o mercado de crédito de carbono; além de possuir flores que são polinizadas por abelhas, é utilizada na medicina popular; as sementes também apresentam potenciais para obtenção da substância galactomanana, que é utilizada como antiviral frente ao HSV1 (vírus Herpes simplex), vírus da febre amarela e dengue (CARVALHO, 1994; CORADIN, 2011).

Mimosa tenuiflora (Willd.) Poir. (jurema-preta) e $M$. ophthalmocentra Mart. ex. Benth. (jurema-branca) são reconhecidas pelo seu potencial econômico, uma vez que suas folhas e frutos são utilizados na alimentação de caprinos, ovinos e bovinos (SILVA, 2013). Algumas espécies de Mimosa são utilizadas em rituais folclóricos, como $M$. artenisiana Heringer \& Paula, com a qual é preparada bebidas alucinógenas em rituais de tribos indígenas (NASCIMENTO et al., 2012; SILVA, 2013).

$\mathrm{Na}$ medicina popular, espécies como Mimosa caesalpiniifolia Benth., Mimosa pudica L e Mimosa scabrella Benth. apresentam usos consideráveis para tratamentos de várias patologias, sendo a primeira para tratar bronquites, estancar sangramentos ou tratar ferimentos, além de ter usos para fins madeireiros, voltados para marcenaria e produção de lenhas, e a segunda para tratamento de difteria, reumatismo e na forma de gargarejo para curar inflamação da garganta, além da utilização das folhas no processo de infusão para colagoga, desobstruente do fígado, amarga, tônica e purgativa, empregada contra icterícia e afecções reumáticas; a infusão das flores na forma de banho é usada para tratamento de tumores e leucorréia; como cataplasma usa-se contra escrófula (LORENZO; MATOS, 2002; COUTINHO, 2009).

O gênero Mimosa está representado por uma diversidade relevante de amostras depositadas no Herbário IAN, abrangendo aproximadamente 683 exsicatas, sendo que o gênero encontra-se totalmente informatizado e cerca de $95 \%$ disponibilizados no Herbário Virtual IAN.

As coleções biológicas têm um papel extremamente importante na representatividade da biodiversidade de um país, intensificando-se pelo processo de degradação das várias formas de vidas, sendo estas testemunhas da biodiversidade e instrumento imprescindível para estudos nas diferentes áreas de conhecimento, principalmente, na sistemática e taxonomia (COSTA et al., 2016).

Nesse contexto, os herbários constituem relevante fonte de informações para diversas áreas, viabilizando estudos de composição original de espécies, recursos genéticos e até mesmo, de espécies raras ou ameaçadas (COSTA et al., 2016). Todo esse processo é possível através de levantamentos em acervos ou bancos de dados dos herbários, podendo-se analisar vários fatores que respondem às mais diversificadas problemáticas das ciências.

A informatização das coleções biológicas tem facilitado todo o processo de levantamento, além de contribuir para a conservação dos dados e melhorar o gerenciamento das informações dos bancos de dados. No Brasil, esse processo 
está em pleno desenvolvimento, com novas ferramentas de estudo no campo de pesquisa botânica, onde todo o processo de informatização dos herbários tem como consequência a universalização e conservação dos dados da biodiversidade vegetal (PEIXOTO, 2005), pois têm sido desenvolvidas e introduzidas com intuito de facilitar e minimizar as atividades inerentes às coleções biológicas.

O Herbário IAN da Embrapa Amazônia Oriental é o terceiro maior da Amazônia e está há mais de sete décadas contribuindo com o manejo e conservação da flora amazônica. Atualmente conta com uma coleção de aproximadamente 195 mil exsicatas, 3 mil tipos nomenclaturais, 8 mil amostras de madeira e 30 mil fotografias de plantas oriundas do herbário de Berlin, além de coleções menores, como flor em meio líquido (321 amostras), plântulas em meio líquido (54), plântulas desidratadas (65), carpoteca com frutos secos (700) e em meio líquido (289), sementes desidratadas (291), e coleções de fungos e Líquens (3.778 amostras) (SOUZA et al., 2013).

Possui um acervo com cerca de $90 \%$ das coleções informatizadas. Além disso, existe um objetivo maior que é disponibilizar os dados no Herbário Virtual IAN, o qual se encontra com aproximadamente 10 mil dados disponíveis no endereço eletrônico (http://brahms.cpatu.embrapa.br/) (SOUZA et al., 2013).

Diante do exposto, o trabalho teve como objetivo realizar o levantamento, informatização e disponibilização dos dados das amostras de Mimosa L. no acervo do herbário IAN da Empresa Brasileira de Pesquisa Agropecuária (EMBRAPA) Amazônia Oriental.

\section{MATERIAL E MÉTODOS}

O trabalho foi implementado em três etapas: levantamento, informatização e disponibilização do gênero Mimosa L.

\section{Levantamento}

Essa etapa teve início com a obtenção dos dados de Mimosa contidas no banco de dados do Herbário IAN. Utilizando-se a ferramenta de busca do sistema BRAHMS (Botanical Reseach and Herbarium Managemet System), extraíram-se os dados do arquivo principal do banco de dados e criou-se um arquivo RDE (Entrada Rápida de Dados) .

A partir do arquivo RDE, os dados das exsicatas foram verificados e comparados com suas respectivas imagens; foram realizadas as verificações da grafia dos nomes científicos e, quando necessário, fez-se a correção nomenclatural por meio de consultas em sites específicos como Lista de Espécies da Flora do Brasil, IPNI (The International Plant Names Index) e Mobot (Missouri Botanical Garden); consultou-se o site do IBGE (Instituto Brasileiro de Geografia e Estatística) para a confirmação ou correção dos nomes de localidades situados dentro do Brasil (Estados, municípios, distritos); também, incluiu-se dados que estavam ausentes no arquivo de RDE.

Como forma de agilizar a busca por informações, as exsicatas receberam códigos de barras, que são facilmente lidas pelo leitor de códigos. Os códigos foram padronizados juntando-se o acrônimo do herbário e o registro da exsicata, por exemplo, IAN123456 e impressos em etiquetas autoadesivas (marca INFORMS, modelo 2850, dimensões 44,45x12,7 mm). Em exsicatas antigas, as etiquetas com códigos de barras, foram coladas próximas ao tombo e coberto por fita mágica Scotch. Em exsicatas novas o código vem impresso na própria etiqueta de coleta.

A digitalização das imagens foi realizada a partir da captura das mesmas, utilizando-se câmera fotográfica semiprofissional de 18 Megapixels, regulada em escala macro (melhor foco), com flash.

Fez-se a edição das imagens com o editor PAINT BRUSH e, posteriormente, renomeou-se as mesmas, utilizando-se recurso de renomeação de imagens disponibilizado pelo programa BRAHMS. Esta renomeação segue os mesmos critérios adotados para fazer o código de barras pelo herbário, Imagens das etiquetas das exsicatas recebem o mesmo tratamento, entretanto, possuem a letra "e" para identificar a presença de etiqueta.

Todas as imagens (Figura 2) foram salvas com a extensão JPG e foram ligadas a seus respectivos dados dentro do arquivo principal do banco de dados.

Figura 1. Registro fotográfico de exsicata de amostra de Mimosa sensitiva L. no acervo do Herbário IAN.

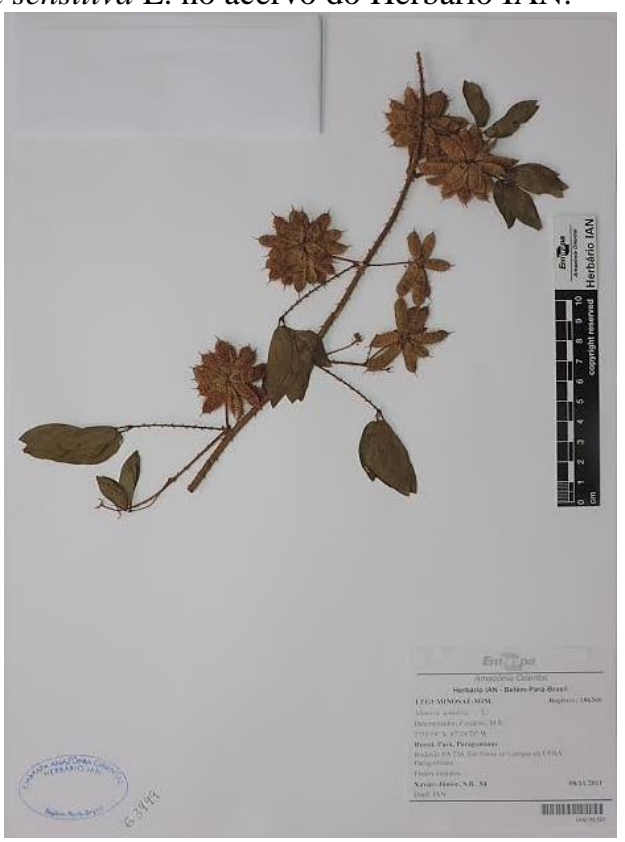

Fonte: Sistema BRAHMS, Herbário IAN, 2016.

\section{Informatização}

Confrontando-se os exemplares do acervo com o arquivo RDE, observou-se que alguns exemplares ainda não possuíam seus dados informatizados, então, os mesmos foram fotografados e seus dados inseridos no arquivo. Também foram feitos registros fotográficos para amostras que já se encontravam em RDE, mas não possuíam imagens. Posteriormente, foi realizada a renomeação de imagens através do BRAHMS e salvas com o número de registro pertencente à exsicata de acordo com os padrões estabelecidos pelo Herbário.

Em seguida, as imagens foram inseridas na biblioteca de imagens do banco de dados do Herbário IAN, ligando o número de registro dos exemplares às suas respectivas imagens.

\section{Disponibilização}

Todo o banco de dados do IAN está armazenado em um servidor torre localizado no Setor de Informática da Embrapa Amazônia Oriental (PC HP, ML 350G6, processador 2.4GHz, 
MemÓria 24GB, Sistema Operacional Windows 2008 Server) de onde saem todos os dados e imagens que estão disponibilizados no herbário virtual. Para que os dados e imagens cheguem até o servidor é necessário usar o componente do BRAHMS chamado de Brahmswebconnect, responsável pela busca dos mesmos no banco de dados, conversão em linguagem de marcação (organizar, separar o conteúdo e agregá-lo a outras linguagens (arquivos xml) (PEREIRA, 2017), finalizando com o produto que é o site do herbário virtual IAN.

As imagens que são disponibilizadas recebem ainda tratamento prévio, sendo as mesmas processadas pelo programa Zoomifyer Enterprise Connect, o qual lhes confere uma maior velocidade ao carregar a imagem.

Figura 2. Disponibilização do gênero Mimosa no Herbário Virtual IAN.

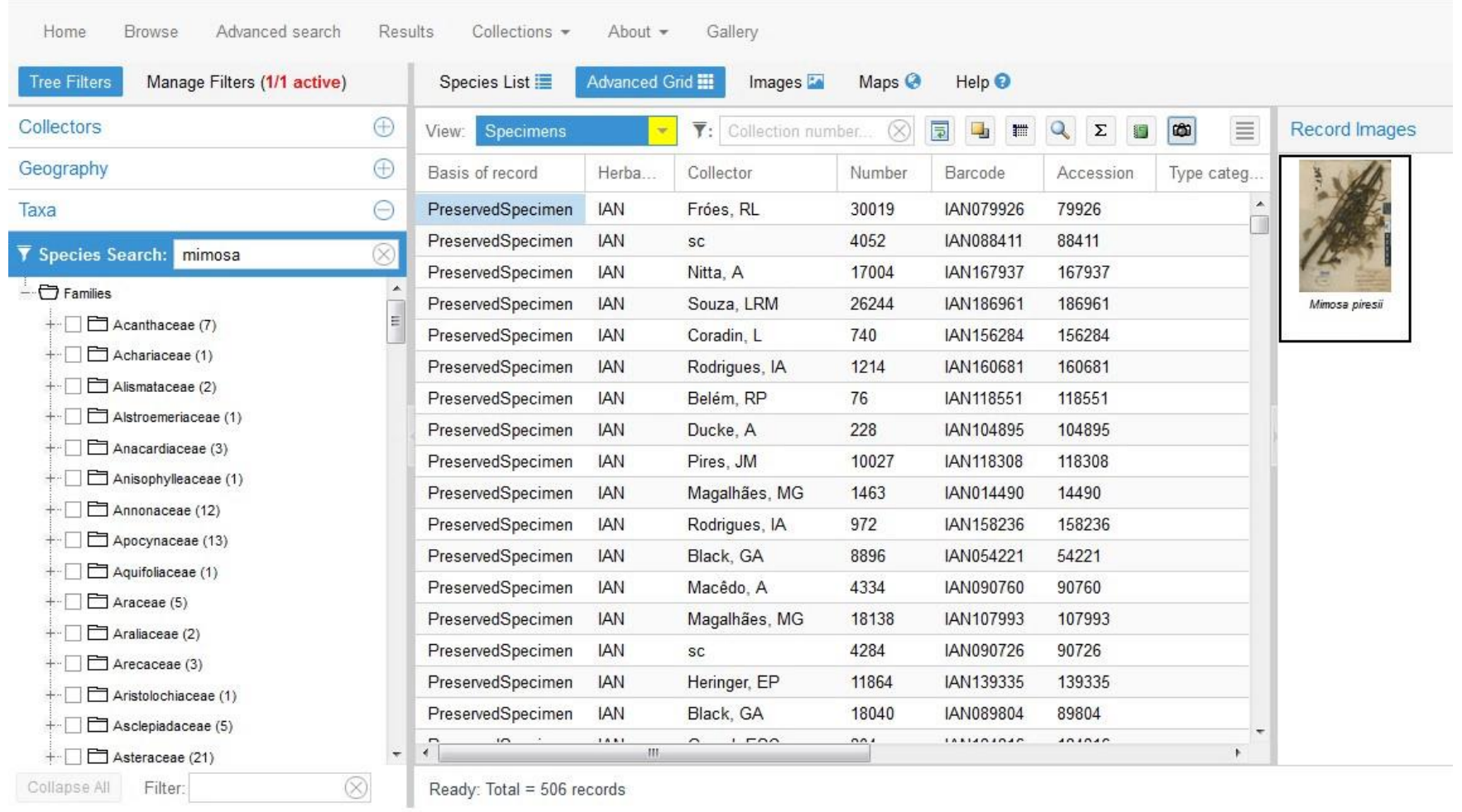

Fonte: Herbário Virtual, 2018

\section{RESULTADOS E DISCUSSÃO}

O gênero Mimosa L. possui 683 espécimes no Herbário IAN, totalizando 176 espécies. Entre as amostras analisadas do acervo, observou-se ampla diversidade de espécies e também grande quantidade de amostras por espécies específicas, conforme a Tabela 1 .

Tabela 1. Espécies de Mimosa presente no Herbário IAN com mais de 5 amostras depositadas no acervo.

\begin{tabular}{lc}
\hline \multicolumn{1}{c}{ ESPÉCIES } & Qt. \\
\hline Mimosa pigra L. & 41 \\
Mimosa pudica L. & 34 \\
Mimosa guilandinae (DC.) Barneby & 21 \\
Mimosa rufescens Benth. & 21 \\
Mimosa somnians Humb. \& Bonpl. ex Willd. & 20 \\
Mimosa camporum Benth. & 19 \\
Mimosa sensitiva L. & 19 \\
Mimosa caesalpiniifolia Benth. & 17 \\
Mimosa myriadenia (Benth.) Benth. & 16 \\
Mimosa quadrivalvis L. & 14 \\
Mimosa xanthocentra Mart. & 14 \\
Mimosa schomburgkii (DC.) Kunth & 13 \\
Mimosa setosa Benth. & 13 \\
Mimosa aurivillus Mart. & 11 \\
Mimosa debilis Humb. \& Bonpl. ex Willd. & 11 \\
Mimosa foliolosa Benth. & 9
\end{tabular}

Mimosa invisa Mart. ex Colla 9

Mimosa pellita Humb. \& Bonpl. ex Willd. 9

Mimosa polydactyla Humb. \& Bonpl. ex Willd. 9

Mimosa acutistipula (Mart.) Benth. 8

Mimosa bimucronata (DC.) Kuntze 8

Mimosa dormiens Humb. \& Bonpl. ex Willd. 8

Mimosa nuda Benth. 8

Mimosa orthocarpa Spruce ex Benth. 8

Mimosa polycarpa Kunth 8

Mimosa arenosa (Willd.) Poit. $\quad 7$

Mimosa velloziana Mart. $\quad 7$

Mimosa barretoi Hoehne 6

Mimosa calodendron Mart. ex Benth. 6

Mimosa gemmulata Barneby 6

Mimosa hirsutissima Mart. $\quad 6$

Mimosa microcephala Humb. \& Bonpl. ex

Willd. 6

Mimosa pithecolobioides Benth. 6

Mimosa scabrella Benth. $\quad 6$

Observou-se que as coletas do gênero Mimosa L. registradas no Herbário IAN foram feitas em vários países da América Latina e também na América do Norte. DUTRA (2009) confirma a ampla distribuição geográfica do gênero, demonstrados pelos dados do IAN, que apresentou $1 \%$ de amostras coletadas na América do Norte e 99\% na América do Sul, em que $83,5 \%$ das amostras de Mimosa foram 
coletadas no Brasil. (Tabela 2), exibindo uma elevada quantidade de locais de ocorrência para este gênero, especialmente, no que tange a região Norte (Figura 3)..

Tabela 2. Países de coleta de amostras de Mimosa depositados no Herbário IAN.

\begin{tabular}{lc}
\hline \multicolumn{1}{c}{ PAÍSES } & Quantidade. \\
\hline Brasil & 626 \\
Venezuela & 9 \\
Argentina & 7 \\
Colômbia & 7 \\
Paraguai & 6 \\
Guiana & 5 \\
Peru & 4 \\
Bolívia & 3 \\
Estados Unidos & 2 \\
Equador & 2 \\
México & 2 \\
Guiana Francesa & 1 \\
Uruguai & 1 \\
Suriname & 1 \\
Sem local definido & 7 \\
\hline
\end{tabular}

O Brasil contém $83,5 \%$ das amostras depositado no Herbário IAN, tendo uma elevada quantidade de locais de ocorrência do gênero. Simon e Proença (2000), afirma que no Brasil há uma estimativa de ocorrência de 340 espécies de Mimosa, sendo que o IAN tem $51,8 \%$ dessas espécies depositadas em seu acervo. Sendo, que as coletas das amostras do território brasileiro é a maior responsável pela representatividade de gênero no acervo do Herbário IAN, especialmente a região norte do país. Portanto, a conservação da flora brasileira, implica diretamente na manutenção dos ecossistemas que as espécies de Mimosa estão inseridas, consequentemente garantindo a preservação das espécies de Mimosa, sendo esta de grande ocorrência no território nacional.

Foi possível observar que o herbário IAN apresenta materiais de coletas de Mimosa abrangendo as cinco regiões brasileiras e diversos habitat, conforme pode-se verificar no Figura 3:

Figura 3. Número de coletas de amostras de Mimosa depositada no Herbário IAN por regiões brasileiras.

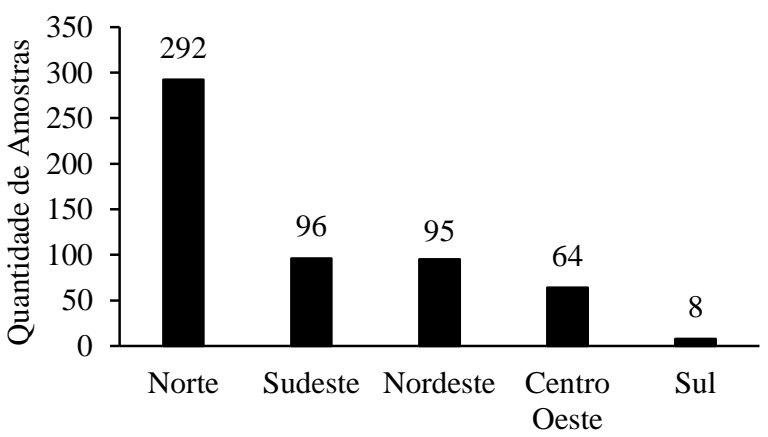

Os coletores que mais contribuíram para acervo de Mimosa L. do Herbário IAN foram Black, G.A. (60 amostras), Magalhães, M.G. (52), Pires, J.M. (44), Ducke, A. (30), Belém, R.P. (26), Simon, M.F. (23), Fróes, R.L. (19), Cordeiro, M.R. (19), Sellow, F. (16), Oliveira, E. (16), Heringer, E.P. (15) e Macêdo, A. (14 amostras) (Tabela 3):

Tabela 3. Quantidade de coletas de Mimosa depositadas no Herbário IAN pelos coletores, por países e estados brasileiros.

\begin{tabular}{|c|c|c|c|c|c|c|c|c|c|c|}
\hline Países & Black & Magalhães & Pires & Ducke & Belém & Simon & Fróes & Cordeiro & Sellow & Oliveira \\
\hline Brasil & 56 & 52 & 44 & 30 & 26 & 23 & 19 & 19 & 15 & 16 \\
\hline Colômbia & 1 & & & & & & & & & \\
\hline Estados Unidos & 2 & & & & & & & & & \\
\hline México & 1 & & & & & & & & & \\
\hline Uruguai & & & & & & & & & 1 & \\
\hline \multicolumn{11}{|c|}{ Locais de coletas por estados brasileiros } \\
\hline Amapá & & & 7 & & & & 3 & 1 & & 1 \\
\hline Amazonas & 3 & & 1 & 14 & & & 7 & 1 & & \\
\hline Bahia & 1 & & & 10 & 13 & & 1 & & & \\
\hline Ceará & 4 & & & & 1 & & & & & \\
\hline Distrito Federal & & 3 & 4 & & 9 & & & & & \\
\hline Espírito Santo & & & & & 1 & & & & & \\
\hline Goiás & & 2 & & & 1 & 2 & & & & \\
\hline Maranhão & 4 & & 4 & & & & 3 & & & \\
\hline Mato Grosso do Sul & & & & & & 1 & & & & \\
\hline Minas Gerais & 7 & 47 & 1 & & 1 & 8 & 1 & & & \\
\hline Pará & 19 & & 24 & 1 & & & 4 & 13 & & 13 \\
\hline Pernambuco & & & & 3 & & & & & & \\
\hline Rondônia & 6 & & 2 & & & 12 & & 4 & & 2 \\
\hline Roraima & 8 & & 1 & 2 & & & & & & \\
\hline São Paulo & 4 & & & & & & & & & \\
\hline Sem estado definido & & & & & & & & & 15 & \\
\hline
\end{tabular}

É notável o número de amostras coletadas na região Norte do país, sendo possível encontrar coletas em seis
Estados da região com uma quantidade apreciável de amostras: Pará (186 amostras), Amazonas (38), Rondônia 
(33), Amapá (17), Roraima (17) e Acre (1 amostra). O Pará apresenta o maior número de amostras, com locais de coletas diversificados em vários municípios paraenses. Ressalta-se que os municípios que mais contribuíram para este resultado foram Belém (52 amostras), Moju (11), Almeirim (9), Monte
Alegre (8), Santarém (7), Itaituba (6), Bragança (6), Salvaterra (6) e Marabá (5 amostras), demostrando uma diversidade de locais coletas botânicas dentro do Estado (Figura 4).

Figura 4. Mapa do Estado do Pará com os municípios que foram coletados as amostras Mimosa depositadas no Herbário IAN.

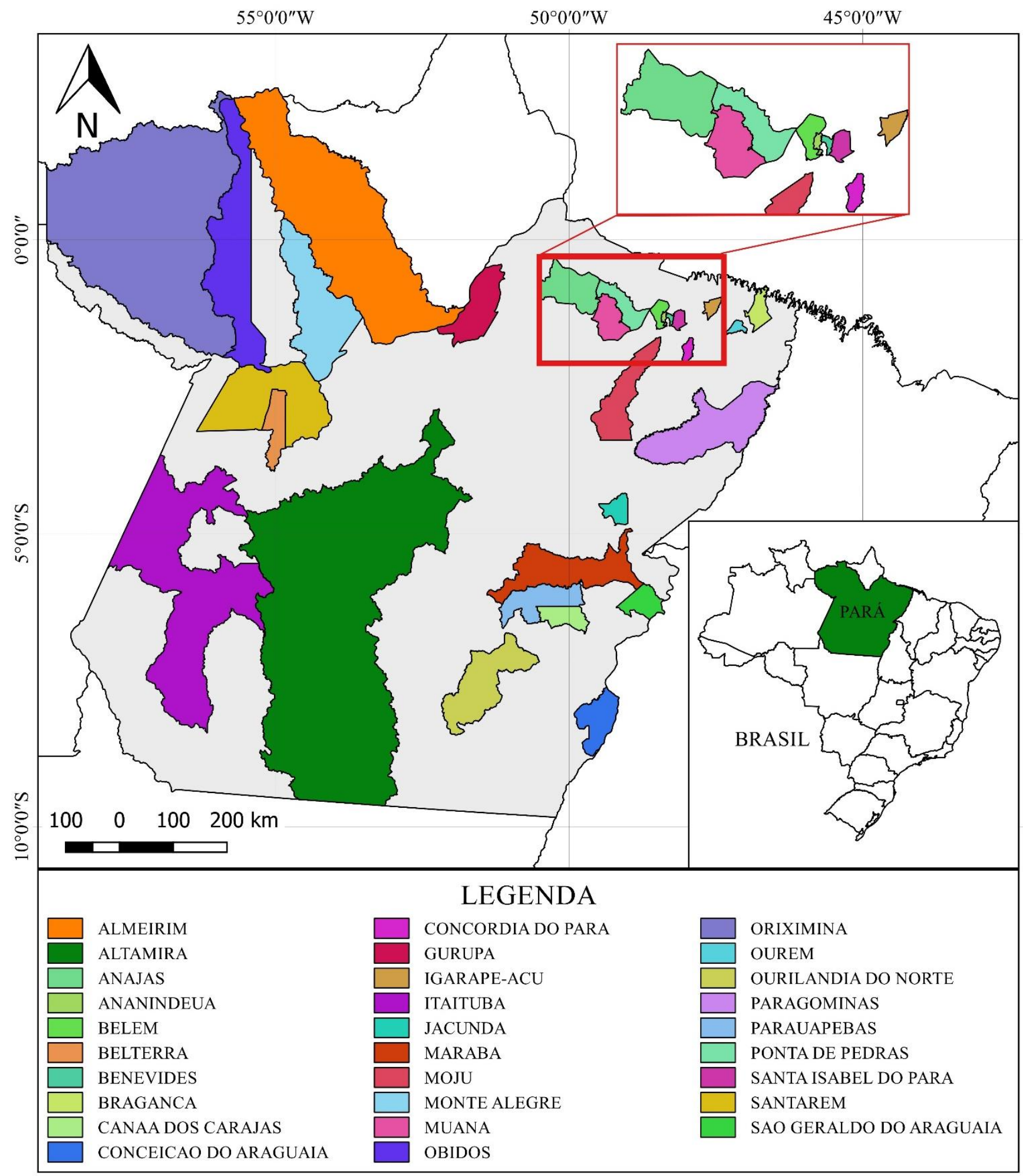

Fonte: Autores, 2018

Foram organizados os 683 exemplares encontrados no acervo, contemplando 176 espécies equivalentes a $34,5 \%$ de espécies que ocorrem no mundo (LEWIS et al., 2005). Todas as informações contidas no banco de dados podem ser consultadas, verificadas e confirmadas através dos registros fotográficos das exsicatas e etiquetas existentes, uma vez que foram obtidos cerca de $93 \%$ das imagens do acervo, 
aumentando a credibilidade da consulta aos dados do IAN (Figura 5).

Figura 5. Proporção de amostras de Mimosa depositada no Herbário IAN com registros fotográficos no Banco de Dados

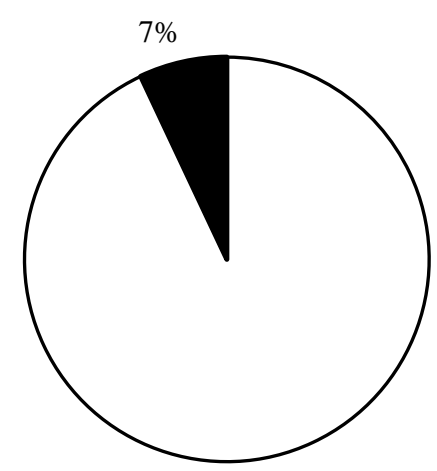

口Amostras com registros fotográficos

- Amostras sem registos fotográficos

$93 \%$

O processo de informatização resultou na verificação e organização dos dados e imagens contidos no banco de dados do herbário IAN, o que culminou com a quantificação do acervo e, com a base devidamente organizada, é possível acessar dados e imagens de toda a coleção de Mimosa usando ferramentas de acesso rápido e prático.

A disponibilização dos dados via internet viabiliza a redução de custos com envio de material para outros herbários; contribui com a preservação das exsicatas; agiliza o processo de identificação e facilita o trabalho desenvolvido por estudantes e pesquisadores de áreas diversas.

Diante da crescente demanda por celeridade na obtenção de informações, o herbário IAN, por meio de sua equipe foi inserido no processo da tecnologia virtual e contribui plenamente quando da divulgação de seus dados. Assim, o acervo de Mimosa, que representa uma pequena parte de todo o herbário foi disponibilizado no herbário virtual IAN (http://brahms.cpatu.embrapa.br/herbario).

A coleção do gênero Mimosa contém 589 espécies disponibilizadas no Herbário Virtual, o que resulta em $86 \%$ de toda coleção disponível. Porém, ainda há ausência de $14 \%$ da coleção no Herbário Virtual, que consiste em 94 amostras não disponibilizadas. A ausência destas é geralmente por estarem emprestadas para outros herbários ou depositadas incorretamente no acervo.

A coleção do gênero Mimosa do Herbário IAN reúne grande quantidade de informações que podem subsidiar estudos e pesquisas nas mais diversas áreas de conhecimento, sendo o processo de levantamento necessário para mensurar todo o acervo presente no herbário e, consequentemente, servindo como fonte de dados para estudos posteriores, que poderão ir de simples estudos básicos até a promoção de políticas públicas ou elaborações de floras.

\section{CONCLUSÕES}

O gênero Mimosa L. encontra-se representado por 683 de amostras no Herbário IAN da Embrapa Amazônia Oriental, com uma vasta diversidade de 176 espécies, com coletas de amostras em diversas localidades da América, caracterizando a relevância do gênero pela sua diversidade nos ambientes naturais e antropizados.

A elevada quantidade de amostras presentes no Herbário IAN indica a diversidade de Mimosa dos locais coletados, especialmente na região norte do Brasil. No entanto, sempre haverá necessidade de se fazer mais coletas botânicas ou intercâmbios com outros herbários, para enriquecimento dos mesmos e melhor entendimento dos grupos taxonômicos, bem como reduzir os vazios de coleta ainda observados para alguns estados brasileiros e, em especial, para o Pará.

No processo de informatização e digitalização, o IAN promove a "alimentação" contínua de dados e imagens de Mimosa no banco de dados, armazenando-os de forma organizada para que também gerações futuras tenham acesso a informações sobre este grupo. $\mathrm{O}$ processo de disponibilização das coleções do IAN torna-se prestigiada pela comunidade científica e pela sociedade comum, pois através da divulgação dessas coleções possibilita-se o alcance a informações da flora amazônica de forma rápida, confiável e segura.

\section{REFERÊNCIAS}

BARNEBY, R. C. Sensitivae censitae: a description of the genus Mimosa Linnaeus (Mimosaceae) in the New World. Mem N Y Bot Gard 65, p. 5-24, 1991

BFG. Growing knowledge: an overview of Seed Plant diversity in Brazil. Rodriguésia, Rio de Janeiro, v.66, n.4, p. 1085-1113, 2015.

BORGES, L. M. Filogenia e sistemática de Mimosa L.: $M$. ser. Pachycarpae Benth. e M. ser. Setosae Barneby. 2014. 269f. Tese (Doutorado em Botânica). Instituto de Biociências, Universidade de São Paulo. 2014.

BORGES, L. M. Mimosoideae na Serra do Cipó, Minas Gerais e análise da variabilidade morfológica de Mimosa macedoana Burkart. 2010. Dissertação (Mestrado em Botânica) - Instituto de Biociências, Universidade de São Paulo, São Paulo, 2010.

CARVAlHO, P. E. R. Sabi, Mimosa caesalpiniifolia. Circular Técnica-Embrapa Florestas, n. 135, 2007. p. 2-7.

CARVALHO, P. E. R. Maricá-Mimosa bimucronata. Circular Técnica-Embrapa Florestas, n. 94, 2004.p.1-6

CARVALHO, P. E. R. Espécies florestais brasileiras: recomendações silviculturais, potencialidades e uso da madeira. Brasil: EMBRAPA-CNPF/SPI, 1994.

CORADIN, L.; SIMINSKI, A.; REIS, A. Espécies nativas da flora brasileira de valor econômico atual ou potencial. Brasília: Ministério do Meio Ambiente, 2011. p. 478 - 493, $656-662$.

COSTA, J. C. M.; LUCAS, F. C. A.; GOIAS, M. A. F.; LEÂO, V. M.; LOBATO, G. J. M. Herbário virtual e universidade: biodiversidade vegetal para ensino, pesquisa e extensão. Scientia Plena, v.12, n.6, 2016.

COUTINHO, A. P. S. Revisão taxonômica e estudos filogenéticos de Mimosa L. sect. Calothamnos Barneby (Leguminosae-Mimosoideae). 2009. 334 f. Tese (Doutorado em Ciências). Universidade de São Paulo. São Paulo. 2009. 
DOURADO, D. A. O.; CONCEIÇÃO, A.S.; SANTOSSILVA, J.. O gênero Mimosa L.(Leguminosae: Mimosoideae) na APA Serra Branca/Raso da Catarina, Bahia, Brasil. Biota Neotropica, Campinas, v.13, n.4, p. 225-240, 2013.

DUTRA, V. F. Diversidade de Mimosa L. (Leguminosae) nos campos rupestres de Minas Gerais: Taxonomia, distribuição geográfica e filogeografia. 2009. 294 f. Tese (Doutorado em Botânica). Universidade Federal de Viçosa. Viçosa. 2009.

LEWIS, G.; SCHRIRE, B.; MACKINDER, B.; LOCK, M. Legumes of the World. Richmond, UK: Royal Botanic Gardens, Kew, 2005. p. 183

LORENZO, H.; MATOS, F. J. A. Plantas medicinais do Brasil: Nativas e exóticas cultivadas. Nova Odessa: Instituto Plantarum, 2002. P. 295.

LPWG - The Legume Phylogeny Working Group. A new subfamily classification of the Leguminosae based on a taxonomically comprehensive phylogeny. Taxon 66. p. 44-77, 2017.

NASCIMENTO, I. A. do; BRAZ-FILHO, R.; CARVALHO, M. G. de; MATHIAS, L.; FONSECA, F. A. Flavonoides e outros compostos isolados de Mimosa artemisiana Heringer e Paula Química Nova, v. 35, n. 11, p. 2159-2164, 2012.

PEIXOTO, F. L. O Processo de Informatização de Herbários: Estudo de Caso. 79f. Dissertação (Mestrado em Botânica) Escola Nacional de Botânica do Instituto de Pesquisas do Jardim Botânico do Rio de Janeiro. Rio de Janeiro, 2005.

PEREIRA, A. P. O que é XML. Disponível: https://www.tecmundo.com.br/programacao/1762-o-que-exml-.htm. Acessado em 21/02/2017.

SILVA, J. S. Filogenia, estudos micromorfológicos e revisão taxonômica de Mimosa ser. Leiocarpa benth. (Leguminosae Mimosoideae). $309 \mathrm{f}$. Tese (Doutorado em Biologia Vegetal). Universidade Estadual de Campinas, Campinas. 2013.

SIMON, M. F.; PROENÇA, C. Phytogeographic patterns of Mimosa (Mimosoideae, Leguminosae) in the Cerrado biome of Brazil: an indicator genus of high-altitude centers of endemism? Biological Conservation, v.96, n.3, p.279-296, 2000.

SOUZA, H. J. R. de; MARTINS-DA-SILVA, R. C. V.; FILER, D. L.; XAVIER-JUNIOR, S. R.; FOURO, A. M. M. Dados do Herbário IAN da Embrapa Amazônia Oriental / Belém, PA: Embrapa Amazônia Oriental, 2013. 43 p.: il.; 15 cm x $21 \mathrm{~cm}$. - (Documentos / Embrapa Amazônia Oriental, ISSN 1983-0513; 392). p. 9 - 10. 